\title{
Influência da intervenção sensório-motora no sistema respiratório de recém-nascidos prematuros
}

\author{
Influence of sensorimotor intervention on the respiratory system of \\ preterm infonts
}

\author{
Natólia Matos Tedesco; Geisy Hellen Mamedes Silva²; Mariane de Oliveira Nunes Reco3; Daniele \\ de Almeida Soares-Marangoni4; Mara Lisiane de Moraes dos Santos ${ }^{4}$; Leila Foerster Merey ${ }^{4}$ \\ 1 Programa de Pós-Graduação Saúde e Desenvolvimento na Região Centro-Oeste. Universidade Federal do Mato Grosso do Sul - \\ UFMS. Campo Grande, MS - Brasil. \\ 2 Universidade Federal do Mato Grosso do Sul - UFMS. Campo Grande, MS - Brasil. \\ 3 Hospital Universitário Maria Aparecida Pedrossian - HUMAP, Universidade Federal do Mato Grosso do Sul - UFMS. Campo \\ Grande, MS - Brasil. \\ 4 Instituto Integrado de Saúde. Universidade Federal do Mato Grosso do Sul - UFMS. Campo Grande, MS - Brasil.
}

Endereço para Correspondência:

Leila Foerster Merey

Rua Antônio Maria Coelho, n. 6681, casa 31, Bairro

Vivendas do Bosque

79021170 - Campo Grande, MS [Brasil]

leilocaf@hotmail.com 


\section{Introdução}

A Organização Mundial de Saúde (OMS) define como recém-nascido pré-termo aquele que nasce com menos de 37 semanas de idade gestacional, independente do peso ao nascer ${ }^{1}$. De todos os nascimentos prematuros no mundo, $60 \%$ concentram-se em determinados países, incluindo o Brasil. Isso torna a prematuridade um importante problema de saúde pública, especialmente quando associada ao baixo peso ao nascer, por ser a principal causa de morbimortalidade no período neonatal ${ }^{2}$. Neste sentido, o cuidado adequado a recém-nascido pré-termo tem sido um dos grandes desafios de saúde no país, sendo crescente a necessidade de medidas para reduzir os índices de mortalidade e morbidade nessa população.

No nascimento prematuro, a imaturidade dos sistemas orgânicos, em especial do sistema respiratório, torna o recém-nascido mais vulnerável a complicações. Além disso, recém-nascidos pré-termo frequentemente permanecem internados em unidades hospitalares neonatais por algum período, onde estão suscetíveis a inúmeros estímulos dolorosos, visuais e sonoros, o que pode desencadear respostas fisiológicas não apropriadas para a idade. Quando o período de internação é prolongado, o estresse constante do ambiente hospitalar e dos procedimentos necessários para manter a vida do recém-nascido, como manipulações, aspiração e ventilação mecânica, podem causar alterações psicológicas e fisiológicas adicionais que agravam seu quadro clínico. Esse estresse pode acarretar em elevação da pressão arterial, aumento do fluxo sanguíneo cerebral e risco de hemorragia, além de perda de peso corporal ${ }^{3}$. Além disso, esse desequilíbrio na fisiologia do organismo pode desencadear queda na saturação de oxigênio e aumento das frequências cardíaca e respiratória ${ }^{4}$, comprometendo o metabolismo global do recém-nascido ${ }^{3}$.

De forma geral, enquanto no período intrauterino o feto encontrava-se em um ambiente aconchegante, com temperatura e luminosidade ideais, ruídos suaves e sem necessidade de es- forço para manter-se vivo, a necessidade abrupta de se adequar ao ambiente extra-uterino da unidade neonatal favorece a desorganização dos sistemas em desenvolvimento, representando prejuízos para a fisiologia e desenvolvimento do recém-nascido ${ }^{5}$ Nesse contexto, como o aumento da sobrevida dos recém-nascidos pré-termo tem se tornado cada vez maior, é preciso que técnicas de intervenção precoce sejam realizadas com a finalidade de minimizar os possíveis atrasos ou complicações aos quais estão suscetíveis.

A fisioterapia tem assumido um papel de grande relevância como potencializadora do cuidado de recém-nascidos pré-termo, tornando-se necessária e rotineira nas unidades de terapia intensiva neonatais (UTIN) e Unidades de Cuidados Intermediários Neonatais (UCIN) mundialmente 6 . Uma das possibilidades de atuação da fisioterapia é a intervenção sensóriomotora precoce, que tem por objetivo apoiar as famílias e potencializar as capacidades do recém-nascido o mais precocemente possível, contribuindo para uma trajetória de desenvolvimento mais saudável para o lactente 7 .

De fato, nos últimos anos, ensaios clínicos e revisões sistemáticas ${ }^{8,9,10}$ apontaram para os efeitos positivos da intervenção sensóriomotora precoce no desenvolvimento de lactentes pré-termo. Ao proporcionar especialmente estímulos táteis e cinestésicos adequados, a intervenção pode aprimorar as funções comportamentais, cognitivas e motoras desses lactentes $8,9,11$. Além disso, a literatura sugere que ao proporcionar melhor organização do sistema postural, a intervenção sensório-motora leva ao melhor funcionamento respiratório em recémnascidos pré-termo hospitalizados ${ }^{12}$. No entanto, considerando que o conhecimento relativo à influência dos estímulos sensório-motores em parâmetros fisiológicos e peso corporal desses recém-nascidos ainda é muito limitado, bem como diante da fragilidade desses recém-nascidos quanto às funções fisiológicas e gasto energético, as contribuições de estudos neste tema tornam-se valiosas. 
Considerando o contexto exposto, o objetivo deste estudo foi verificar a influência de um protocolo de intervenção sensório-motora sobre parâmetros fisiológicos e peso corporal em recém-nascidos pré-termo estáveis internados em unidade de cuidados intermediários.

\section{Métodos}

Trata-se de um estudo preliminar de intervenção não controlada, realizado no Hospital Regional de Mato Grosso do Sul, Campo Grande, MS, no período de março a julho de 2015.

Inicialmente, foram incluídos 50 recémnascido pré-termos internados em unidades de cuidados intermediários, com idade gestacional entre 28 a 36 semanas e 6 dias, estáveis hemodinamicamente, com mais de 72 horas de vida, em respiração espontânea, sem malformações congênitas e sem hemorragia peri-intraventricular (HPIV) grau III e/ou IV, com peso mínimo de 1100 gramas. A grande parcela de recém-nascidos internados dentro desse serviço hospitalar apresentava HPIV grau I ou II, sendo, então, incluídos na pesquisa. Foram excluídos 20 recémnascidos pré-termo, por motivos de: alta hospitalar antes do término da coleta, desistência dos responsáveis, perda de peso durante os dias de intervenção, ou manipulação no período de 15 minutos após o término da intervenção. Ao final, portanto, a amostra foi constituída por 30 RNPT. O estudo foi aprovado pelo Comitê de Ética em Pesquisa em Seres Humanos da Universidade Federal de Mato Grosso do Sul (UFMS) (parecer $\mathrm{n}^{\circ}$. 1008856), seguindo as Diretrizes e Normas Regulamentadoras das Pesquisas Envolvendo Seres Humanos (Resolução 466/2012) do Conselho Nacional de Saúde. Os pais/responsáveis leram e assinaram o termo de consentimento livre e esclarecido.

O protocolo de intervenção sensório-motora foi executado pela mesma fisioterapeuta, no período vespertino, após 60 minutos da dieta do recém-nascido, por 3 dias consecutivos. O protocolo continha os seguintes procedimentos terapêuticos: 1) dissociação passiva de tronco, 2) chutes e alcance alternados passivos, 3) estímulo tátil na cabeça utilizando as próprias mãos do recém-nascido, 4) estímulo tátil na face utilizando as próprias mãos do recém-nascido, e 5) posicionamento em decúbito dorsal mimetizando o padrão flexor fisiológico. Os procedimentos foram realizados na mesma sequência em todos os participantes, com duração de aproximadamente 2-2,5 minutos para cada procedimento. Desta forma, o protocolo diário durava cerca de 10 minutos.

As respostas à intervenção foram mensuradas por meio da mensuração das seguintes variáveis: frequência cardíaca (FC); frequência respiratória (FR); saturação de oxigênio $\left(\mathrm{SaO}_{2}\right)$; Boletim de Silverman-Andersen (BSA); e peso corporal. As variáveis $\mathrm{FC}$ e $\mathrm{SaO}_{2}$ foram coletadas por meio do monitor de oxímetro de pulso (modelo Dixtal DX2022), disponível no leito de cada recém-nascido. A FR foi mensurada considerando-se o número de movimentos da caixa torácica ao longo de um minuto. Para o BSA, a pesquisadora foi treinada para mensuração e quantificação considerando os sinais físicos de esforço respiratório. O peso corporal foi mensurado pela equipe de enfermagem por meio de balança digital durante o período matutino. Não houve recém-nascido que estivesse utilizando cateter de oxigênio durante o experimento.

As variáveis fisiológicas foram mensuradas intra-sessão nos seguintes momentos: 1) imediatamente antes da intervenção, 2) $\log 0$ após o término da intervenção, e 3) 15 minutos após o término. Entre os momentos 1 e 2 as mães e profissionais da equipe eram orientados a não manusear o recém-nascido, exceto nos casos onde se fizesse de necessário, para preservar a integridade do recém-nascido.

Para a análise estatística, os efeitos do dia de intervenção (entre sessões: dia 1, dia 2, dia 3) e do momento de avaliação (intra-sessão: antes da intervenção, após, 15 minutos após), bem como a interação entre estes fatores para as variáveis $\mathrm{FC}, \mathrm{FR}$, e $\mathrm{SaO}_{2}$, foi aplicado teste ANOVA de duas vias de medidas repetitivas, seguido 
pelo pós-teste de Tukey. Os mesmos testes foram aplicados para a comparação entre os dias para a variável peso corporal. Para as comparações entre os dias e entre os momentos de avaliação para o BSA, foi aplicado o teste de Friedman seguido pelo pós-teste de Dunn. A análise estatística foi realizada por meio do programa estatístico SigmaPlot, versão 12.5, considerando um nível de significância de $5 \%$.

\section{Resultados}

Foram avaliados 30 recém-nascidos prétermo, com idade gestacional de 232,43 $\pm 3,14$ dias (média \pm erro padrão).

Os recém-nascidos apresentaram ganho significativo de peso corporal no decorrer dos três dias $\left(\mathrm{F}_{2,29}=33,55 ; \mathrm{p}<0,001\right.$; poder do teste $=1,00)$, sendo que a cada dia houve ganho significativo em relação ao dia anterior $(\mathrm{p}<0,05)$. No primeiro dia do estudo, o peso médio dos recém-nascidos foi de 1955,27士84,10 gramas, enquanto no segundo e no terceiro dia o peso foi de $1994,00 \pm 84,07$ e $2014,60 \pm 83,47$ gramas, respectivamente.

Na Tabela 1 estão apresentados os resultados referentes à $\mathrm{FC}$, $\mathrm{FR} \mathrm{e} \mathrm{SaO}_{2}$, de acordo com o dia de análise e o momento de avaliação.

Em relação à $\mathrm{FC}$, na análise geral dos dados não houve efeito significativo do dia $\left(\mathrm{F}_{2,29}=3,06 ; \mathrm{p}=0,601\right.$; poder do teste $\left.=0,39\right)$, do momento de avaliação $\left(\mathrm{F}_{2,29}=0,51 ; \mathrm{p}=0,054\right.$; poder do teste $=0,05)$ e nem interação entre estes fatores $\left(\mathrm{F}_{4,29}=0,97 ; \mathrm{p}=0,422 ;\right.$ poder do teste $\left.=0,05\right)$; ou seja, a FC não se alterou significativamente com o dia avaliado e o tratamento realizado. Estes resultados estão ilustrados na Figura 1.

Em relação à $F R$, houve um efeito significativo do dia $\left(\mathrm{F}_{2,29}=41,2 ; \mathrm{p}=0,005\right.$; poder do teste $=1,00)$ e do momento de avaliação $\left(F_{2,29}=5,88\right.$; $\mathrm{p}<0,001$; poder do teste $=0,79)$ entretanto, não houve interação entre estes fatores $\left(\mathrm{F}_{4,29}=1,50\right.$; $\mathrm{p}=0,207$; poder do teste $=0,16$ ). De forma geral, a FR no momento logo após a intervenção foi maior do que aquela nos momentos antes e 15 minutos
Tabela l: Valores (média \pm erro padrão) referentes ò frequência cardíaca (FC), à frequência respiratória $(\mathrm{FR})$ e ò saturação de oxigênio sanguíneo $\left(\mathrm{SaO}_{2}\right)$, de acordo com o dia de intervenção e o momento em relação ao tratamento. Valor do $p$ do dia. Bpm: batimentos por minuto; ir: incursões respiratórias

\begin{tabular}{cccc}
\hline & \multicolumn{3}{c}{ Momento } \\
\cline { 2 - 4 } Variável & Antes & Logo após & $\begin{array}{c}15 \text { minutos } \\
\text { após }\end{array}$ \\
\hline FC (bpm) & & & \\
Dia 1 & $146,17 \pm 3,93$ & $145,10 \pm 3,54$ & $144,30 \pm 3,42$ \\
Dia 2 & $144,40 \pm 2,59$ & $150,30 \pm 3,22$ & $145,13 \pm 2,71$ \\
Dia 3 & $146,20 \pm 3,63$ & $150,70 \pm 2,74$ & $146,63 \pm 2,58$ \\
Valor de $p$ & 0,601 & & \\
\hline FR (ir) & & & \\
Dia 1 & $52,97 \pm 1,75$ & $58,97 \pm 1,72$ & $47,53 \pm 1,77$ \\
Dia 2 & $50,73 \pm 1,62$ & $57,10 \pm 1,58$ & $47,07 \pm 1,51$ \\
Dia 3 & $47,97 \pm 1,50$ & $51,93 \pm 1,52$ & $45,20 \pm 1,33$ \\
Valor de p & 0,005 & & \\
\hline SaO (\%) & & & \\
Dia 1 & $95,33 \pm 0,71$ & $96,77 \pm 0,56$ & $96,73 \pm 0,58$ \\
Dia 2 & $97,00 \pm 0,54$ & $97,00 \pm 0,49$ & $96,83 \pm 0,43$ \\
Dia 3 & $96,67 \pm 0,50$ & $97,80 \pm 0,43$ & $97,60 \pm 0,45$ \\
Valor de p & 0,108 & & \\
\hline
\end{tabular}

Fonte: própria.

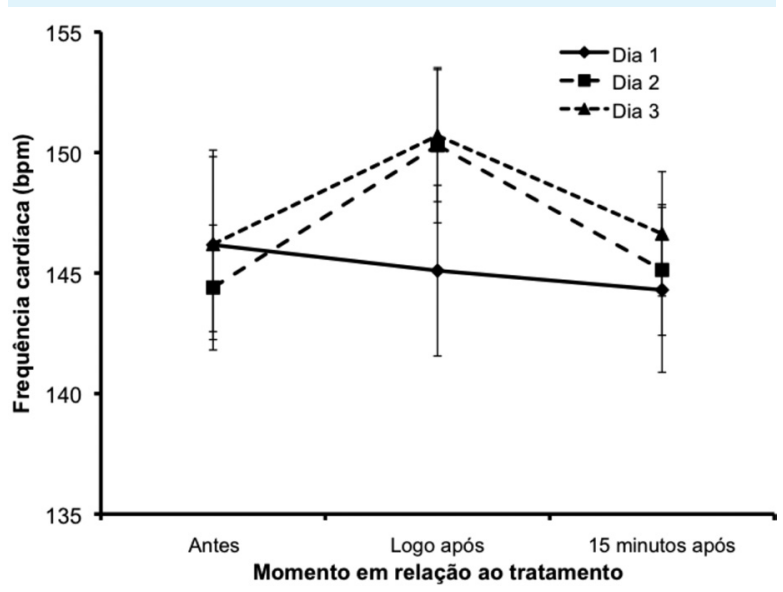

Figura 1: Frequência cardíaca dos recémnascidos avaliados de acordo com o dia de intervenção e o momento em relação ao tratamento (momento de avaliação)

Cada símbolo representa a média e a barra o erro padrão. Bpm: batimentos por minuto.

Fonte: própria. 
após $(\mathrm{p}<0,05)$. Por outro lado, a FR no momento 15 minutos após a intervenção foi menor do que a observada antes da intervenção $(p<0,05)$; ou seja, a intervenção levou a um aumento da FR inicial, seguida de uma queda significativa após 15 minutos do tratamento. Além disso, de forma geral, a FR no terceiro dia foi menor do que a observada no primeiro dia $(\mathrm{p}<0,05)$. Estes resultados estão ilustrados na Figura 2.

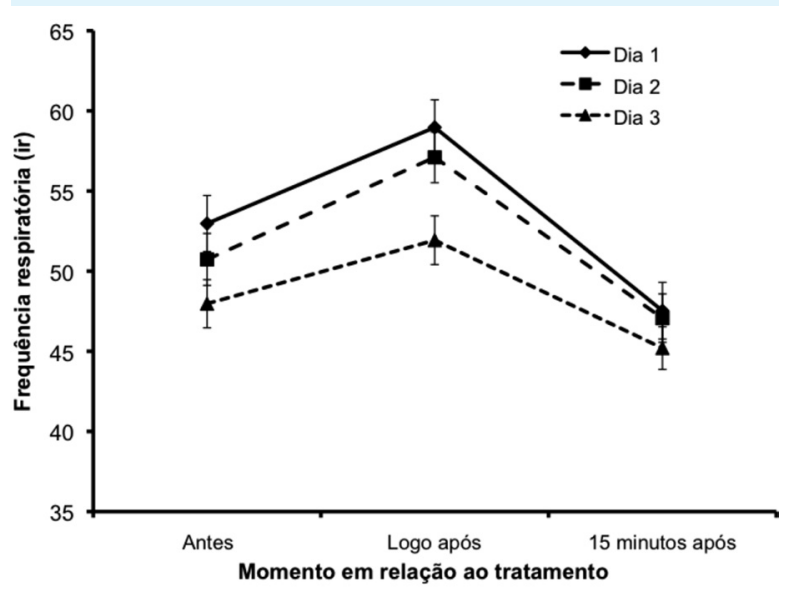

Figura 2: Frequência respiratória dos recém-nascidos avaliados de acordo com o dia de intervenção e o momento em relação ao tratamento (momento de avaliação) Cada símbolo representa a média e a barra o erro padrão. Ir: incursões por minuto.

Fonte: própria.

Quanto à $\mathrm{SaO}_{2}$, foi observado apenas efeito significativo do momento de avaliação $\mathrm{F}_{2,29}=2,31$; efeito do dia: $\mathrm{p}=0,108$; poder do teste $=0,26$; efeito do momento: $\mathrm{F}_{2,29}=4,38 ; \mathrm{p}=0,017$, poder do teste $=0,61$; interação entre os fatores: $F_{4,29}=1,51$; $p=0,203$, poder do teste $=0,16$, sendo que, de forma geral, a $\mathrm{SaO}_{2}$ no momento logo após a intervenção foi significativamente maior do que aquela observada no momento antes da intervenção $(p<0,05)$. Estes resultados estão ilustrados na Figura3.

Os resultados referentes ao BSA estão apresentados na Tabela 2. Apesar de ser observada uma diferença significativa entre os momentos de avaliação no segundo e no terceiro dia na análise geral dos dados (dia 1: $p=0,002$; dia 2: $p=0,005)$, não houve diferença entre os momen-

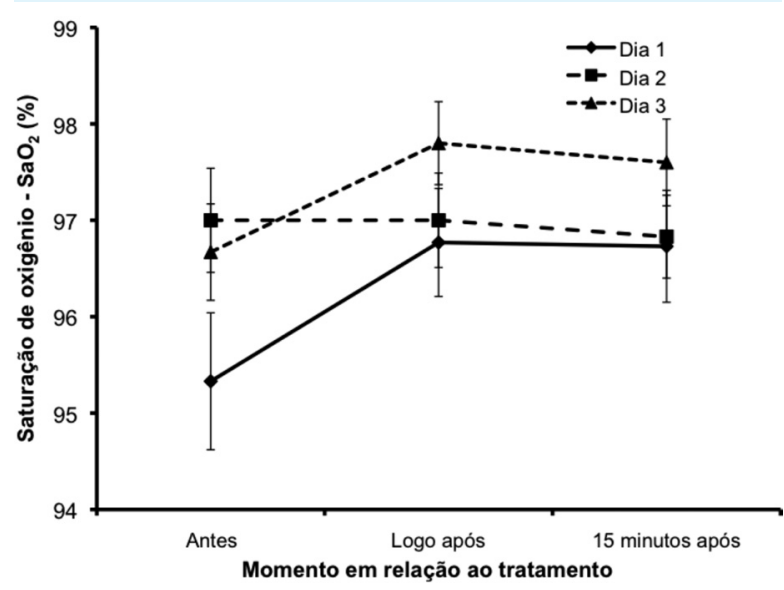

Figura 3: Saturação de oxigênio dos recémnascidos avaliados de acordo com o dia de intervenção e o momento em relação ao tratamento (momento de avaliação) Cada símbolo representa a média e a barra o erro padrão.

Fonte: própria.

tos na comparação dois a dois pelo pós-teste de Dunn ( $p>0,05)$. Além disso, não houve diferença entre os momentos no primeiro dia $(p=0,208)$ e nem entre os dias em nenhum dos momentos avaliados (antes: $\mathrm{p}=0,721$; logo após: $\mathrm{p}=0,441 ; 15$ minutos após: 0,156).

\section{Discussão}

O presente estudo investigou a influência de uma intervenção sensório-motora em parâmetros fisiológicos e no ganho de peso corporal de recém-nascido pré-termo hospitalizados. De forma geral, observou-se constância na maioria dos parâmetros fisiológicos estudados.

Analisando o peso corporal, observamos que o mesmo aumentou ao longo do estudo, sugerindo que o gasto energético utilizado pelos recém-nascidos durante a intervenção não causou perda de peso. Costa et a ${ }^{13}$ sugerem que a intervenção sensório-motora não compromete o ganho de peso de recém-nascidos pré-termo, corroborando nosso achado. O estudo realizado por Diego et al ${ }^{14}$, onde investigaram os efeitos de uma estimulação tátil e cinestésica em recém-nascidos pré-termo e demonstraram que apenas os recém-nascidos 
Tabela 2: Valores(mediana, mínimo e máximo) referentes ao Boletim de Silverman-Adersen (BSA), de acordo com o dia de intervenção e o momento em relação ao tratamento (momento de avaliação)

\begin{tabular}{|c|c|c|c|c|}
\hline \multirow{2}{*}{$\begin{array}{c}\text { BSA } \\
\text { (o a } 5 \text { pontos) }\end{array}$} & \multicolumn{3}{|c|}{ Momento } & \multirow{2}{*}{ Friedman; $p$} \\
\hline & Antes & Logo após & 15 minutos após & \\
\hline Dia 1 & $0(0 \text { e } 2)^{A a}$ & $0(0 \text { e } 2)^{A a}$ & $0(0 \text { e } 2)^{\mathrm{Aa}}$ & $\left(X_{2}^{2}=3,14\right) ; 0,208$ \\
\hline Dia 2 & $0(0 \text { e } 3)^{A a}$ & $0(0 \text { e } 3)^{A a}$ & $0(0 \text { e } 1)^{\mathrm{Aa}}$ & $\left(X_{2}^{2}=12,61\right) ; 0,002$ \\
\hline Dia 3 & $0(0 \text { e } 2)^{A a}$ & $0(0 \text { e } 3)^{\text {Aa }}$ & $0(0 \text { e } 1)^{\mathrm{Aa}}$ & $\left(X_{2}^{2}=10,65\right) ; 0,005$ \\
\hline Friedman; $p$ & $\left(X_{2}^{2}=0,66\right) ; 0,721$ & $\left(X_{2}^{2}=1,64\right) ; 0,441$ & $\left(X_{2}^{2}=3,71\right) ; 0,156$ & \\
\hline
\end{tabular}

Valor de $p$ no teste de Friedman com pós-teste de Dunn. Mesmas letras maiúsculas na linha indicam que não há diferença significativa entre os momentos de análise. Mesmas letras minúsculas na coluna indicam que não há diferença significativa em relação aos dias de análise (pós-teste de Dunn, p>0,05).
Fonte: própria.

aca do que recém-nascidos de termo, porque ainda não possuem balanço dos sistemas simpático-parassimpático bem estabelecidos. Isto pode explicar a ausência de alterações significativas na frequência cardíaca no presente estudo. De acordo com Guinsburg ${ }^{16}$, o estresse pode causar desequilíbrio na fisiologia do or-

que receberam estímulos de pressão moderada, em comparação com pressão leve, apresentaram maior ganho de peso e aumento da atividade vagal cardíaca. Os autores sugeriram que o ganho de peso após estimulação tátil e cinestésica pode ser mediada por aumento da atividade vagal. A estimulação pode ativar as fibras eferentes vagais que inervam o sistema digestivo, estimulando barorreceptores e mecanorreceptores dentro da pele. $\mathrm{Ou}$ seja, pode-se especular que efeitos no ganho de peso em recém-nascidos pré-termo podem ser mediados pelo aumento da motilidade gástrica que foi estimulada por meio da estimulação tátil e cinestésica. Porém, não podemos afirmar que o ganho de peso observado ao longo do presente estudo seja decorrente da intervenção sensório-motora realizada, pois a maioria dos recém-nascidos fazia uso de sonda nasogástrica, apresentaram mudanças de dietas ou realizavam terapias fonoaudiólogas no decorrer da internação. Desta forma, não é possível associar diretamente o ganho de peso com a intervenção aplicada.

A frequência cardíaca não apresentou efeitos significativos quanto ao momento de avaliação, sugerindo que a intervenção sensóriomotora não apresentou sobrecarga ao coração. Segundo Selig et $\mathrm{al}^{15}$, a variabilidade da frequência cardíaca pode caracterizar a capacidade de adaptação do organismo frente a estímulos externos. Esses autores sugerem que recém-nascidos pré-termo exibem comportamento menos complexo da variabilidade da frequência cardí- ganismo e desencadear aumento da frequência cardíaca. Desta forma, como não encontramos alterações significativas nesta variável, inferimos que a intervenção sensório-motora aplicada se mostrou uma técnica que não proporcionou estímulos estressantes aos recém-nascidos.

Em relação à frequência respiratória, houve diferença significativa logo após a intervenção, sendo maior nesse momento, onde o recémnascido acabava de receber estímulos, do que no momento anterior e nos 15 minutos após o término. Portanto, o protocolo de intervenção sensório-motora parece interferir imediatamente no sistema respiratório do recém-nascido. Por outro lado, aos 15 minutos após o término da intervenção, a frequência respiratória sofreu queda em relação ao momento antes da intervenção. Isto sugere um efeito positivo não imediato sobre a mecânica respiratória.

O recém-nascido pré-termo apresenta diferenças importantes em relação ao controle da respiração e frequência respiratória em comparação, por exemplo, ao adulto. O recém-nascido pré-termo, além de não possuir uma boa coordenação entre seus músculos respiratórios, é mais sensível aos estímulos periféricos no que se refere ao controle respiratório. Isto pode explicar os resultados encontrados no presente estudo quanto ao aumento imediato da frequência respiratória após a intervenção sensório-motora, possivelmente aumentando a profundidade da respiração. No entanto, com o posicionamen- 
to do recém-nascido em padrão flexor ao final da intervenção, permanecendo acomodado ao longo dos 15 minutos seguintes, pode ter ocasionado otimização da mecânica respiratória, repercutindo na diminuição da frequência respiratória. Segundo Fucile et al ${ }^{12}$, melhor organização no sistema postural talvez favoreça o funcionamento respiratório. Pode-se sugerir, ainda, que uma melhora na mecânica respiratória pode ter contribuído para um menor gasto energético nos recém-nascidos e, consequentemente, favorecido o ganho de peso corporal observado.

Além do próprio organismo do recém-nascido, a frequência respiratória sofre alterações de acordo com o ambiente externo em que o recémnascido se encontra. Por exemplo, Fernández et $\mathrm{al}^{17}$ demonstraram que a luz e a temperatura ambiental são fatores que influenciam no ritmo circadiano e ultradiano da frequência respiratória. Esses autores recomendam que as interpretações de variações dessa variável levem em consideração o horário das análises. Como no presente estudo as avaliações foram realizadas sempre no período vespertino, acreditamos que as condições ambientais relacionadas ao horário não influenciaram nossos resultados quanto à frequência cardíaca.

A saturação de oxigênio apresentou diferença apenas no momento logo após a intervenção, apresentando um aumento, quando comparado com o momento anterior. Olmedo et $\mathrm{al}^{18} \mathrm{e}$ Almeida et $\mathrm{al}^{19}$, ao utilizaram o posicionamento mãe-canguru, onde o bebê permanece em contato com a pele materna posicionado em padrão flexor, também observaram um aumento da saturação de oxigênio logo após a aplicação do método. Os autores inferiram que o método proporcionou relaxamento psicofisiológico do recém-nascido, o que provavelmente levou à diminuição do consumo de oxigênio e melhora da oxigenação tecidual. No presente estudo, como houve queda da frequência respiratória logo após a intervenção sensório-motora, seguida de melhora 15 minutos após, não é possível afirmar se a técnica acarretou relaxamento nos recémnascidos. Porém, possivelmente não houve me- lhora da saturação de oxigênio nesse período dos 15 minutos porque os recém-nascidos já haviam atingido valores ótimos dessa variável no momento logo após a intervenção.

Segundo Silverman e Andersen ${ }^{20}$, o BSA é um método clínico útil para quantificar o grau de desconforto respiratório e estimar a gravidade do comprometimento pulmonar. Ao observarmos os valores do BSA no presente estudo, verificamos que os recém-nascidos iniciaram e permaneceram ao longo do experimento sem sinais de esforço respiratório. Isto sinaliza que a intervenção não acarretou prejuízos ao sistema respiratório dos recém-nascidos pré-termo, podendo-se considerar essa não variação no BSA como ponto favorável. Concordamos com Souza et $\mathrm{al}^{21}$, que não encontraram alterações no BSA em técnicas de fisioterapia respiratória em recém-nascidos pré-termo em unidade neonatal, quanto à pouca discussão na literatura sobre a avaliação de alterações no BSA, sendo necessário expandir as pesquisas com este parâmetro, uma vez que o mesmo auxilia na percepção preventiva do desconforto respiratório durante as intervenções em unidades neonatais.

Os resultados deste estudo podem não ser generalizáveis a outras populações de recémnascidos pré-termo hospitalizados. Além disso, é preciso cautela quanto às interpretações dos resultados, uma vez que não houve grupo controle. Vale também destacar as limitações durante o período de coleta, onde houve a perda de 20 recém-nascidos devido a condições como alta hospitalar, comum em estudos em recém-nascidos hospitalizados. No entanto, é importante salientar que apesar das dificuldades frente às rotinas de trabalho, torna-se importante o esforço coletivo no cuidado multidisciplinar nas unidades neonatais para uma melhor integralidade do cuidado dos recém-nascidos, assim como novos ensaios clínicos randomizados controlados relacionados aos efeitos de intervenções sensóriomotora nessa população, são de grande relevância, permitindo ampliar os achados do presente estudo, potencializando a prática baseada em evidências na área. 


\section{Conclusõo}

A intervenção sensório-motora proporcionou melhoras na frequência respiratória e na saturação de oxigênio. Além disso, os demais parâmetros estudados, como frequência cardíaca, Boletim de Silverman-Andersen e peso corporal, não sofreram prejuízos. Portanto, acreditamos que o protocolo utilizado apresentou potencial benéfico e debaixo risco ao recém-nascido pré-termo envolvidos. De modo geral, o estudo agrega conhecimento preliminar sobre uma técnica cujos efeitos ainda são pouco esclarecidos na população estudada.

\section{Referências}

1. WHO: Recommended definition terminology and format for statistical tables related to the perinatal period and rise of a new certification for cause of perinatal deaths. Modifications recommended by FIGO as amended, 1976. Acta Obstet Gynecol Scand. 1977;56: 247-253.

2. March of Dimes, PMNCH, Save the Children, World Health Organization. Born Too Soon: the Global Action Report on Preterm Birth. World Health Organization. Geneva, 2012.

3. Souza MWCR, Silva WCR, Araújo SAN. Quantification of manipulations in neonatal intensive care unit: proposal of protocol elaboration. ConScientiae saúde. 2008;7(2):269-274.

4. Da Silva CM, Cação JMR, Silva KCS, Marques CF, Merey LSF. Respostas fisiológicas de recém-nascidos pré-termo submetidos à musicoterapia clássica. Rev. paul. pediatr. 2013;31(1):30-6.

5. Perini C, Seixas MC, Catão ACSM, Silva GD, Almeida VS, Matos PBC. Banho de ofurô em recém-nascidos no alojamento conjunto: um relato de experiência. Rev. pesqui. cuid. fundam. (Online). 2014;6(2):785-792.

6. Nicolau CM, Falcão MC. Efeitos da fisioterapia respiratória em recém-nascidos: análise crítica da literatura. Rev. paul. pediatr. 2007;25:72-5.

7. Formiga CKMR, Pedrazzani ES, Tudela E. Intervenção precoce com bebês de risco. Rio de Janeiro: Atheneu; 2010.

8. Sgandurra G, Lorentzen J, Inguaggiato E, Bartalena L, Beani1 E, Cecchi F, et al. A randomized clinical trial in preterm infants on the effects of a home-based early intervention with the 'CareToy System'. PLoS ONE. 2017; 12(3): e0173521.
9. Spittle A, Orton J, Anderson P, Boyd R, Doyle LW. Early developmental intervention programmes posthospital discharge to prevent motor and cognitive impairments in preterm infants. Cochrane Database Syst Rev. 2012; 12:CD005495

10. Pepino VC, Mezzacappa MA. Application of tactile/ kinesthetic stimulation in preterm infants: a systematic review. J.pediatr. (Rio J). 2015;91:213-33.

11. Formiga CK, Pedrazzani ES, Silva FPS, Lima CD. Eficácia de um programa de intervenção precoce com bebês pré-termo. Paidéia. 2004; 14(29): 301-311.

12. Fucile S, McFarland DH, Gisel EG, Lau C. Oral and nonoral sensorimotor interventions facilitate suckswallow-respiration functions and their coordination in preterm infants. Early Hum Dev. 2012;88(6):345-50.

13. Costa PP, Ruedell AM, Weinmann ARM, Keske SM. Influência da estimulação sensório-motora-oral em recém-nascidos pré-termo. Rev. CEFAC. 2011; 3(4):599-606

14. Diego MA, Field T, Hernandez-Reif M. Vagal activity, gastric motility, and weight gain in massaged preterm neonates. The Journal of Pediatrics. 2005; 47: 50-5.

15. Selig FA, Tonolli ER, Silva EVCM, Godoy MF. Variabilidade da Frequência Cardíaca em Neonatos Prematuros e de Termo.Arq. bras. cardiol. 2011;96(6):443-449.

16. Guinsburg R. Dor no recém-nascido: importância do estudo da dor no recém-nascido. Manual de neonatologia. Rio de Janeiro: Revinter. 2000.

17. Fernández JA, Llano JM, Gómez JA, Ramos MA, Casado ML, Suárez JG. Biologic rhythm of respiratory rate in the first trimester of life. An EspPediatr. 1992;37:377-82

18. Olmedo MD, GabasGS, Merey LSF, SouzaLS, Muller KTC; dos Santos MLM, et al. Respostas fisiológicas de recém-nascidos pré-termo submetidos ao Método Mãe-Canguru e a posição prona. Fisioterpesqui. 2012;19(2):115-121.

19. Almeida CM, Almeida AFN, Forti EMP. Efeitos do Método Mãe Canguru nos sinais vitais de recémnascidos pré-termo de baixo peso. Rev. bras. fisioter. 2007;11(1):1-5.

20. Silverman, WA, Andersen, DH. A controlled clinical trial of effects of water mist on obstructive respiratory signs, death rate and necropsy findings among premature infants. Pediatrics. 1956;17(1-10).

21. Souza JAQ, Moran CA. Fisioterapia respiratória em recém-nascidos pré-termo: ensaio clínico randomizado. Pediatr.mod. 2013;49(11):434-43. 